\title{
Differences in Clinical and Imaging Presentation of Maxillary sinus fungus ball with and without Intralesional Hyperdensity
}

\author{
Pei-wen $\mathrm{Wu}^{1}$, Ta-Jen Lee ${ }^{1}$, Shih-Wei Yang ${ }^{2}$, Yen-Lin Huang ${ }^{1}$, Yun-Shien Lee ${ }^{2}$, Che-Fang \\ $\mathrm{Ho}^{1}$, and Chien-Chia Huang ${ }^{1}$ \\ ${ }^{1}$ Chang Gung Memorial Hospital and Chang Gung University \\ ${ }^{2}$ Chang Gung Memorial Hospital
}

January 3, 2021

\begin{abstract}
Objectives: Maxillary sinus fungal balls (MSFBs) mostly occur in older individuals and demonstrate female predominance. Early diagnosis is important to avoid treatment delays. This study aimed to elucidate the differences in clinical and imaging presentation of MSFB with and without intralesional hyperdensity (IH). Design and setting: This was a retrospective review in a tertiary medical center. We retrospectively identified 588 patients underwent endoscopic sinus surgery procedures for MSFB between 2005 and 2018. The clinical characteristics and computed tomography (CT) findings including the presence of complete or partial opacification, IH, and odontogenic pathology were reviewed. Participants: 588 MSFB patients Results: Patients with unilateral MSFB had a mean age of 57.4 years and demonstrated female predominance (64.63\%). The overall female-to-male ratio was highest at 51-60 years (2.02) and rose to 2.60 in MSFB with IH only. Compared to those with IH, MSFB without IH was significantly more common in males $(\mathrm{OR}=2.496 ; \mathrm{P}<.0001)$, in those with diabetes mellitus (DM) $(\mathrm{OR}=2.094 ; \mathrm{P}=.010)$ and adjacent maxillary odontogenic pathology $(\mathrm{OR}=1.697 ; \mathrm{P}=.030)$. Complete opacification on $\mathrm{CT}$ was less common in MSFB without IH $(\mathrm{OR}=0.617 ; \mathrm{P}=.025)$. Conclusion: The highest female-to-male ratio for MSFB at 51-60 years corresponded to the age of menopause. Patients with MSFB without IH were more likely to have DM, no female predominance, adjacent maxillary odontogenic pathology, and partial opacification of the sinus, compared to those with IH. These clinical features may aid earlier identification of MSFB without IH.
\end{abstract}

\begin{abstract}
Objectives: Maxillary sinus fungal balls (MSFBs) mostly occur in older individuals and demonstrate female predominance. Early diagnosis is important to avoid treatment delays. This study aimed to elucidate the differences in clinical and imaging presentation of MSFB with and without intralesional hyperdensity (IH).

Design and setting: This was a retrospective review in a tertiary medical center. We retrospectively identified 588 patients underwent endoscopic sinus surgery procedures for MSFB between 2005 and 2018. The clinical characteristics and computed tomography (CT) findings including the presence of complete or partial opacification, IH, and odontogenic pathology were reviewed.
\end{abstract}

Participants: 588 MSFB patients

Results: Patients with unilateral MSFB had a mean age of 57.4 years and demonstrated female predominance $(64.63 \%)$. The overall female-to-male ratio was highest at 51-60 years (2.02) and rose to 2.60 in MSFB with IH only. Compared to those with IH, MSFB without IH was significantly more common in males (OR $=2.496 ; \mathrm{P}<.0001)$, in those with diabetes mellitus $(\mathrm{DM})(\mathrm{OR}=2.094 ; \mathrm{P}=.010)$ and adjacent maxillary odontogenic pathology $(\mathrm{OR}=1.697 ; \mathrm{P}=.030)$. Complete opacification on $\mathrm{CT}$ was less common in MSFB without IH $(\mathrm{OR}=0.617 ; \mathrm{P}=.025)$. 
Conclusion: The highest female-to-male ratio for MSFB at 51-60 years corresponded to the age of menopause. Patients with MSFB without IH were more likely to have DM, no female predominance, adjacent maxillary odontogenic pathology, and partial opacification of the sinus, compared to those with IH. These clinical features may aid earlier identification of MSFB without IH.

\section{Key points:}

1. Female predominance was seen in the maxillary sinus fungal ball (MSFB), especially in the 51-60 years' group, corresponding to the age of menopause.

2. Female predominance was not observed in the patients with MSFB without intralesional hyperdensity $(\mathrm{IH})$ on computed tomography $(\mathrm{CT})$ scan images.

3. Patients with MSFB without IH on CT scan images were more likely to have diabetes mellitus, adjacent maxillary odontogenic pathologies, and partial opacification of the sinus compared to those with IH.

Keywords: Fungus ball, Intralesional hyperdensity, Maxillary sinus, Odontogenic pathology, Rhinosinusitis

\section{INTRODUCTION}

Fungi are recognized as etiologic agents in a wide range of disease states of the nose and paranasal sinuses. The International Society for Human and Animal Mycology divides fungal rhinosinusitis (FRS) into invasive and non-invasive types based on histopathologic evidence of fungus penetrating host tissue. ${ }^{1}$ The sinus fungal ball (SFB), usually found in the maxillary sinus (MSFB), is the most frequently encountered form of noninvasive FRS in clinical practice. ${ }^{1}$ Several studies have reported an increasing incidence of SFB over the past few decades in Asia. ${ }^{2-5}$ Yoon et al. reported annual incidence of SFB remained below $5 \%$ until 2001, but increased to over $10 \%$ since 2011 in Korea. ${ }^{4}$ Liu et al. found out the incidence of SFB in the last 5 years was significantly greater than that in the first 5 years during the 10-year study period (2008-2017) in China. ${ }^{5}$ Studies have also shown that SFB mostly occurs in older individuals, with a female predominance. The average age at presentation has been reported to be 49 to 61.1 years, with women accounting for $60.1 \%$ to $76.7 \%$ of $^{\text {cases. }}{ }^{4-8}$

In addition, an increasing number of researchers have suggested that adjacent odontogenic infection increases the risk of MSFB owing to the close relationship between the antral teeth and sinus floor. ${ }^{9}$ Furthermore, endodontic treatment on maxillary teeth is considered to be a significant risk factor for the development of MSFB. ${ }^{10}$ The sinus mucosa may be damaged by chemical and physical trauma as a result of endodontic treatment, causing inflammation. Since maxillary odontogenic pathologies and endodontic procedures increase in frequency with aging, the role of odontogenic etiologies in MSFB may explain the predominance of this condition in the older population. ${ }^{6,11}$

Endoscopic sinus surgery (ESS) for eradicating SFB usually achieves good outcomes and has been considered as the standard treatment. ${ }^{7}$ Therefore, early diagnosis of SFB is important to avoid unnecessary medical therapy and treatment delays. Intralesional hyperdensity $(\mathrm{IH})$, included calcifications with a nodular or linear shape, indicate the presence of heavy metal deposition within fungal hyphae ${ }^{12,13}$ and has been the most specific characteristic of SFB on computed tomography $(\mathrm{CT})$ scan images. In the literature, the prevalence of $\mathrm{IH}$ on $\mathrm{CT}$ images of MSFB ranges from $66 \%$ to $82 \% .5,8,14$ However, for those without IH on CT scan images, the diagnosis of MSFB continues to be a challenge. Our previous study proposed an algorithm to improve the identification of MSFB based on the findings of pre-operative CT scans. ${ }^{15}$ In this study, we retrospectively investigated patients with SFB who underwent ESS in our institute between 2005 and 2018. The aims of this study were to elucidate the clinical characteristics of MSFB and to increase pre-operative identification of the presence of MSFB, especially in those without IH on CT scan images.

\section{MATERIALS AND METHODS}

\section{Participants and setting}

Patients who underwent ESS for CRS and SFB were identified using automated and manual chart reviews, as described below. We conducted an automated search of the histopathology database of our institute 
between 2005 and 2018. We then manually reviewed the chart records of the identified patients to confirm the study groups. A total of 6195 ESS procedures were identified during the study period; 713 patients with SFB were selected from the database based on the exclusion criteria, which included patients with allergic fungal rhinosinusitis or invasive FRS (Figure 1). In order to evaluate the incidence of SFB, the ratio of the number of ESS procedures for SFBs to the total ESS procedures was calculated for each year.

Since clinical features differ according to the location of the SFB, and unilateral MSFB constituted the majority ${ }^{16}, 592$ patients with unilateral MSFB were identified for analysis. Among them, CT scan images of four patients were not available for review; we therefore evaluated the CT findings of 588 patients for the presence of complete or partial opacification and manifestations of IH. Odontogenic etiology was determined based on the clinical history and CT findings of adjacent maxillary odontogenic lesions including periapical lucency, periodontal bone loss, oroantral fistula, and exogenous dental reconstructive/restorative materials (Figure 2). ${ }^{17}$

\section{Statistical methods}

Statistical analysis was performed with MATLAB 2015b program (MathWorks Inc., Natick, MA, U.S.A.). Patients with MSFB were divided into two groups based on whether they presented IH on CT images; clinical characteristics and CT findings were compared between groups. Univariate analysis of categorical variables was performed using the $\chi^{2}$ test or Fisher's exact test, where appropriate. Univariate analysis of continuous variables was performed using the Mann-Whitney U test. Logistic regression analysis was performed to evaluate the associations of multiple different variables with MSFB without IH. For each significant independent variable, the odds ratio (OR) and 95\% confidence interval were calculated. A Pvalue of $<.05$ was considered to be statistically significant.

\section{RESULTS}

During the study period, the overall percentage of ESS procedures for SFB in our institute was 11.58\% $(713 / 6159)$. The annual incidence of SFB demonstrated an increasing trend since 2005, increased to over $10 \%$ since 2008. Twenty-five $(3.51 \%)$ patients with SFB had bilateral lesions. Among the unilateral FBs, single sinus involvement was observed in $679(98.69 \%)$ cases; the maxillary sinus was involved in the majority of cases (86.05\%). The distribution of sinus involvement by FBs is shown in Figure 1 .

The clinical characteristics of the 592 patients with unilateral MSFB are shown in Table 1 . The mean age of patients was $57.4( \pm 13.53)$ (range: 18 to 90$)$ years. Female predominance was seen in our series, with 380 female patients $(64.63 \%)$ and 208 males $(35.37 \%)$; 115 (19.56\%) patients had comorbid medical conditions that may weaken the immune system, including diabetes mellitus (DM), liver cirrhosis, autoimmune disorders, end-stage renal disease, and bronchial asthma. Among them, DM was the most common comorbidity $(14.80 \%)$. Recurrence was seen in $11(1.87 \%)$ patients; $7(1.19 \%)$ cases of complicated FB including facial cellulitis and orbital complications were observed. Fungal culture was performed in 211 cases (35.64\%); among them, 29 (13.74\%) showed positive results. The main species (14/29, 48.28\%) was Aspergillus.

On the CT scan images, 386 cases $(65.65 \%)$ showed complete opacification of the maxillary sinus, 462 cases $(78.57 \%)$ had IH, and 119 cases $(20.24 \%)$ had adjacent maxillary odontogenic pathology. The clinical characteristics of MSFB with and without IH were compared to evaluate the relationship between IH on CT, odontogenic pathology, and the degree of opacification; the results have been presented in Table 2 . Multivariate logistic regression analysis showed complete opacification on CT was less common in MSFB without IH $(\mathrm{OR}=0.617 ; \mathrm{P}=.025)$. Compared to those with $\mathrm{IH}, \mathrm{MSFB}$ without IH was significantly more common in males $(\mathrm{OR}=2.496 ; \mathrm{P}<.0001)$, in those with diabetes mellitus $(\mathrm{DM})(\mathrm{OR}=2.094 ; \mathrm{P}=.010)$ and adjacent maxillary odontogenic pathology $(\mathrm{OR}=1.697 ; \mathrm{P}=.030)$ (Table 3) .

The female-to-male ratio in each decade of age was further analyzed in the two study groups; the results have been shown in Figure 3. Female predominance was seen in all MSFB cases. The overall female-to-male ratio was highest at 51-60 years (2.02) and the ratio rose to 2.60 in the MSFB with IH group in the same age range. In contrast, female predominance was not seen in the MSFB without IH group. The difference 
between the 51-60 and 61-70 years groups was most significant.

\section{DISCUSSION}

In this cohort, the female-to-male ratio was highest in the 51-60 years' group, corresponding to the age of menopause. This may implicate the association of post-menopausal hormonal changes and the formation of SFB, and explain the female predominance, especially in the elderly. Additionally, patients with MSFB without IH on CT images were more likely to have DM, adjacent maxillary odontogenic pathologies, and partial opacification of the sinus compared to those with $\mathrm{IH}$; female predominance was not seen in this patient group.

Studies have shown that SFB mostly occurs in older individuals and has a female predominance. ${ }^{5-8}$. However, there is no consensus on the explanation for this phenomenon. Our study focused on unilateral MSFB; the impact of age and sex were analyzed. We observed that the female-to-male ratio was highest at 51-60 years of age (2.02). The mean age (SD) at menopause in Taiwan is $50.2( \pm 4.0)$ years. ${ }^{18}$ This suggests that postmenopausal hormonal changes may be associated with the formation of SFB. Although the precise mechanism is not clear, the nasal mucosa is affected by changes in female sex hormones. ${ }^{19}$ Özler et al. and Gumussoy et al. both reported on the prolongation of nasal mucociliary clearance times in menopausal women. ${ }^{20,21}$ Impaired mucociliary activity may weaken the defense mechanism of the nasal respiratory epithelium, resulting in failed clearance of airborne fungal spores and formation of SPB.

However, female predominance was not seen in the MSFB without IH group on CT scan images. In this group, patients were more likely to have DM and adjacent maxillary odontogenic pathologies. We hypothesized that both these factors contributed more directly to impair sinonasal ciliary function and increase risk of local infection and/or inflammation in the maxillary sinus than sex hormones; the gender-related difference was therefore diminished in this group.

The SFB is the most common FRS, but its incidence in the general population is unknown. Recent studies have shown that the incidence of SFB is increasing since the mid-2000s. ${ }^{2-5}$ In this study, we analyzed the data of 713 patients with SFB treated at a single medical center between 2005 to 2018. In 2005, the proportion of SFB cases in the total number of cases of ESS was only $5.5 \%$, but exceeded $10 \%$ since 2008. Although these results may not represent the exact annual incidence of SFB in Taiwan, they have reflected an increasing tendency. Improved awareness of the disease, improved diagnostic tools and techniques, broad-spectrum antibiotic use, and aging of the population may have contributed to this phenomenon. ${ }^{5}$ Among these causes, we speculated that the extensive use of CT scans may have been the most important factor. National health insurance pays in full for indicated CT scans in Taiwan since 1995. The database shows that the utilization of CT scans in Taiwan has increased between 1997 and 2008. ${ }^{22}$ In addition, the popularity of dental implants increased the possibility of discovering asymptomatic MFSB during the pre-procedure evaluation.

In the current study, patients with MSFB without IH on CT scan images were more likely to have DM and adjacent maxillary odontogenic pathologies. Both these clinical features contributed to increased susceptibility to secondary bacterial infections. The clinical symptoms may be more severe, resulting in timely visits to healthcare facilities. The early diagnosis of SFB in this study group may have corresponded to the relatively high proportion of partial opacification of sinuses on CT scan images. We may consider patients with MSFB without IH on CT scan images to have early-stage MSFB. Short disease duration and inadequate fungal metabolic metal deposition resulted in partial opacification of sinuses without IH on CT scan images.

Unlike invasive FRS, SFB usually occurred in immune competent patients. The relationship between SFB and chronic disease has not been confirmed. ${ }^{16}$ In our study population, the prevalence of DM in MSFB patients was $14.8 \%$, which is higher than the prevalence of $8.35 \%$ (2005-2008) and 9.1\% (2015-2018), respectively, in the general population in Taiwan. ${ }^{23}$ This observation suggests that there may be a correlation between DM and the occurrence of SFB. Hyperglycemic acidosis may impair oxidative and non-oxidative mechanisms of phagocyte fungal clearance, a major component of innate human immunity. ${ }^{24}$ Altered microvascularization of the nasal mucosa in patients with DM also results in decreased mucociliary clearance. ${ }^{25}$ Post-operative strict glycemic control must be instituted to prevent recurrence. 
Aspergillus species are the most frequently encountered organisms in MSFB, only based on histological evidence. ${ }^{11}$ However, only few studies have evaluated fungal cultures of MSFB because of the low culturepositive rates resulting from poor viability of the fungal hyphae. Liu et al. collected 669 samples from SFB for microbial cultivation, and fungi were discovered in 151 (22.6\%) samples. Among them, Aspergillus spp. $(72.8 \%)$ was the most prevalent fungal species. ${ }^{5}$ In our study, fungal culture was performed in 211 MSFB cases (171 with IH and 40 without $\mathrm{IH}$ ); it yielded a $13.74 \%$ positivity rate. Aspergillus spp. was the most dominant species (13 in IH group and 1 in non-IH group); however, there was no significant difference in the Aspergillus culture-positivity rate between these two groups. The correlation between the fungal species of MSFB and IH on CT scan images remains unclear. Future studies utilizing next-generation sequencing for identifying fungal species may help clarify the exact mechanism.

This study has several limitations that warrant consideration. First, we only enrolled patients who underwent ESS for SFB; this may have introduced some degree of selection bias. Second, this study had a retrospective case-control design. We defined patients with adjacent odontogenic infection based on CT findings and related medical records; we could not determine cases with endodontic treatment and the disease course of the dental problem. Third, information regarding microbiology was not available for most patients in this study; different species of fungi may demonstrate different CT imaging features. A large-scale prospective study is thus needed for further information.

\section{CONCLUSION}

In this cohort, the highest female-to-male ratio for MSFB at 51-60 years corresponded to the age of menopause. Patients with MSFB without IH were more likely to have DM, no female predominance, adjacent maxillary odontogenic pathology, and partial opacification of the sinus, compared to those with IH. These clinical features may aid earlier identification of MSFB without IH.

\section{REFERENCES}

1. A Chakrabarti, DW Denning, BJ Ferguson, et al. Fungal rhinosinusitis: a categorization and definitional schema addressing current controversies. Laryngoscope. 2009;119:1809-1818.

2. JS Kim, SS So, SH Kwon. The increasing incidence of paranasal sinus fungus ball: a retrospective cohort study in two hundred forty-five patients for fifteen years. Clin Otolaryngol. 2017;42(1):175-179.

3. JS Lee, SY Shin, KH Lee, SW Kim, JS Cho. Change of prevalence and clinical aspects of fungal ball according to temporal difference. Eur Arch Otorhinolaryngol. 2013;270(5):1673-1677.

4. YH Yoon, J Xu, SK Park, JH Heo, YM Kim, KS Rha. A retrospective analysis of 538 sinonasal fungus ball cases treated at a single tertiary medical center in Korea (1996-2015). Int Forum Allergy Rhinol. 2017;7(11):1070-1075.

5. X Liu, C Liu, H Wei, et al. A retrospective analysis of 1,717 paranasal sinus fungus ball cases from 2008 to 2017. Laryngoscope. 2020;130(1):75-79.

6. P Nicolai, D Lombardi, D Tomenzoli, et al. Fungus ball of the paranasal sinuses: experience in 160 patients treated with endoscopic surgery. Laryngoscope. 2009;119(11):2275-2279.

7. GJ Ledderose, T Braun, CS Betz, K Stelter, A Leunig. Functional endoscopic surgery of paranasal fungus ball: clinical outcome, patient benefit and health-related quality of life. Eur Arch Otorhinolaryngol. 2012;269(10):2203-2208.

8. Chen JC, Ho CY. The significance of computed tomographic findings in the diagnosis of fungus ball in the paranasal sinuses. Am J Rhinol Allergy. 2012;26(2):117-119.

9. PV Tomazic, E Dostal, M Magyar, et al. Potential correlations of dentogenic factors to the development of clinically verified fungus balls: a retrospective computed tomography-based analysis. Laryngoscope. 2016;126(1):39-43.

10. GY Park, HY Kim, JY Min, HJ Dhong, SK Chung. Endodontic treatment: a significant risk factor for the development of maxillary fungal ball. Clin Exp Otorhinolaryngol. 2010;3(3):136-40.

11. F Pagella, E Matti, FD Bernardi, et al. Paranasal sinus fungus ball: diagnosis and management. Mycoses. 2007;50(6):451-456.

12. Z Jiang, K Zhang, W Huang, Q Yuan. A preliminary study on sinus fungus ball with microCT and 
x-ray fluorescence technique. PLoS One. 2016;11(3):e0148515.

13. P Nicolai, M Mensi, F Marsili, et al. Maxillary fungus ball: zinc-oxide endodontic materials as a risk factor. Acta Otorhinolaryngol Ital. 2015;35(2):93-6.

14. H Cha, Y Song, YJ Bae, et al. Clinical Characteristics Other Than Intralesional Hyperdensity May Increase the Preoperative Diagnostic Accuracy of Maxillary Sinus Fungal Ball. Clin Exp Otorhinolaryngol. 2020;13(2):157-163.

15. CF Ho, TJ Lee, PW Wu, et al. Diagnosis of a maxillary sinus fungus ball without intralesional hyperdensity on computed tomography. Laryngoscope. 2019;129(5):1041-1045.

16. P Grosjean, R Weber. Fungus balls of the paranasal sinuses: a review. Eur Arch Otorhinolaryngol. 2007;264(5):461-70.

17. A Whyte, R Boeddinghaus. Imaging of odontogenic sinusitis. Clin Radiol. 2019;74(7):503-516.

18. TY Shen, C Strong, T Yu. Age at menopause and mortality in Taiwan: A cohort analysis. Maturitas. 2020;136:42-48.

19. A Haeggström, B Ostberg, P Stjerna, P Graf, H Hallén. Nasal mucosal swelling and reactivity during a menstrual cycle. ORL J Otorhinolaryngol Relat Spec. 2000;62(1):39-42.

20. GS Özler, E Akbay, AN Akkoca, OS Karapınar, GÖ Şimşek. Does menopause efect nasal mucociliary clearance time? Eur Arch Otorhinolaryngol 2015;272(2):363-366.

21. S Gumussoy, M Gumussoy, I Hortu, AM Ergenoglu. The effect of surgical menopause after bilateral oophorectomy on hormonal changes, mucociliary clearance, and quality of life. Eur Arch Otorhinolaryngol. 2020;277(10):2793-2800.

22. HY Tsai, CJ Tung, CC Yu, YS Tyan. Survey of computed tomography scanners in Taiwan: dose descriptors, dose guidance levels, and effective doses. Med Phys 2007;34(4):1234-1243.

23. Health Promotion Administration, Ministry of Health and Welfare, Taiwan. Survey on the Prevalence of Hypertension, Hyperglycemia and Hyperlipidemia in Taiwan. Accessed Sep. 7, 2020.

24. RY Chinn, RD Diamond. Generation of chemotactic factors by Rhizopus oryzae in the presence and absence of serum: relationship to hyphal damage mediated by human neutrophils and effects of hyperglycemia and ketoacidosis. Infect Immun. 1982;38(3):1123-1129.

25. M Müller, S Betlejewski. Nasal mucosa in patients with diabetes mellitus. Otolaryngol Pol. 2003;57(3):361-364.

Table 1. Presentation of demographic, clinical, and therapeutic data of the patients examined.

\begin{tabular}{ll}
\hline variable & Descriptive statistics \\
\hline Case number & 592 \\
Age, year (mean $\pm \mathrm{SD})$ & $57.44( \pm 13.53)$ \\
Gender & \\
Male & $208(35.37 \%)$ \\
Female & $380(64.63 \%)$ \\
Odontogenic & $119(20.24 \%)$ \\
Underlying diseases & \\
Asthma & $21(3.57 \%)$ \\
DM & $87(14.80 \%)$ \\
ESRD & $9(1.53 \%)$ \\
Liver cirrhosis & $5(0.85 \%)$ \\
Autoimmune disease & $7(1.19 \%)$ \\
S/P maxilla surgery & $10(1.70 \%)$ \\
S/P maxillary dental implant & $15(2.55 \%)$ \\
NPC s/p CCRT & $3(0.51 \%)$ \\
Previous sinonasal cancer s/p CCRT & $3(0.51 \%)$ \\
Concomitant maxillary tumor & $4(0.68 \%)$ \\
Extrasinus complication & $7(1.19 \%)$ \\
Recurrence & $11(1.87 \%)$
\end{tabular}




\begin{tabular}{ll}
\hline variable & Descriptive statistics \\
\hline CT image & $462(78.57 \%)$ \\
Calcified spots & $386(65.65 \%)$ \\
Complete opacities & $6.7( \pm 1.8)$ \\
Laboratory data & $29(13.74 \%)$ \\
WBC (1000/uL) & Positive fungus culture ${ }^{+}$ \\
\hline
\end{tabular}

DM, Diabetes mellitus; ESRD, End stage renal disease; S/P, Status post; NPC, Nasopharyngeal carcinoma; CCRT, Concurrent chemoradiotherapy

+ Fungal culture was performed in 211 cases.

Table 2. Comparison of clinical characteristics between maxillary sinus fungal ball with and without intralesional hyperdensity on preoperative computed tomography scan images.

\begin{tabular}{lllll}
\hline & $\begin{array}{l}\text { MSFB with IH } \\
(\mathrm{n}=462)\end{array}$ & $\begin{array}{l}\text { MSFB Without IH } \\
(\mathrm{n}=126)\end{array}$ & $\mathrm{P} \mathrm{value}^{+}$ & $95 \% \mathrm{CI}$ \\
\hline Age (years) & $57.68 \pm 13.49$ & $56.53 \pm 13.69$ & 0.398 & $0.979-1.008$ \\
Gender (male) & $142 / 462(30.74 \%)$ & $66 / 126(52.38 \%)$ & $<0.001^{*}$ & $1.66-3.70$ \\
Odontogenic & $85 / 462(18.40 \%)$ & $34 / 126(26.98 \%)$ & $0.045^{*}$ & $1.04-2.59$ \\
$\begin{array}{l}\text { Opacification } \\
\text { (complete) }\end{array}$ & $313 / 462(67.75 \%)$ & $73 / 126(57.94 \%)$ & $0.045^{*}$ & $0.44-0.98$ \\
DM & & & & \\
Asthma & $61 / 462(13.20 \%)$ & $26 / 126(20.63 \%)$ & $0.047^{*}$ & $1.03-2.84$ \\
ESRD & $17 / 462(3.68 \%)$ & $4 / 126(3.17 \%)$ & 1.000 & $0.28-2.60$ \\
\hline
\end{tabular}

MSFB, maxillary sinus fungal ball; IH, intralesional hyperdensity; CI, confidence interval; DM, Diabetes mellitus; ESRD, end stage renal disease.

Data are represented as mean \pm stand deviation.

$* \mathrm{P}<.05$

+Mann-Whitney $U$ test for continuous variables; $\chi^{2}$ test and Fisher's exact test for categorical variables.

Table 3. Multivariate logistic regression analysis of the clinical characteristics related to maxillary sinus fungal ball without intralesional hyperdensity

\begin{tabular}{lllllll}
\hline Variable & Odds ratio & $95 \%$ CI & $\beta$ & SE $(\beta)$ & Wald $\chi^{2}$ & P value \\
\hline Age (years) & 0.993 & $0.978-1.009$ & 0.007 & 0.008 & 0.724 & 0.395 \\
Gender (male) & 2.496 & $1.658-3.758$ & -0.915 & 0.209 & 19.216 & $<0.0001^{*}$ \\
Odontogenic & 1.697 & $1.051-2.738$ & -0.529 & 0.244 & 4.690 & $0.030^{*}$ \\
Opacification (complete) & 0.617 & $0.404-0.942$ & 0.483 & 0.216 & 5.013 & $0.025^{*}$ \\
DM & 2.094 & $1.195-3.670$ & -0.739 & 0.286 & 6.665 & $0.010^{*}$ \\
Asthma & 0.798 & $0.247-2.571$ & 0.226 & 0.597 & 0.143 & 0.705 \\
ESRD & 0.444 & $0.049-4.045$ & 0.812 & 1.127 & 0.519 & 0.471 \\
\hline
\end{tabular}

CI, confidence interval; DM, Diabetes mellitus; ESRD, end stage renal disease. 
$* \mathrm{P}<.05$

\section{FIGURE LEGENDS}

Figure 1. Algorithm for identifying study cohorts.

Figure 2. Computed tomographic features of maxillary sinus fungus ball with intralesional hyperdensity (A), without intralesional hyperdensity (B), with periodontal bone loss $(\mathrm{C})$, with exogenous dental filling material (D), with periapical lucency (E), and with penetrating dental implant $(\mathrm{F})$.

Figure 3. Female-to-male ratio in each decade of age. Female predominance was seen in the maxillary sinus fungus ball (MSFB) cases, overall. The female-to-male ratio was highest at 51-60 years (2.02) and rose to 2.60 in cases of MSFB with intralesional hyperdensity $(\mathrm{IH})$ in the same range of age. In contrast, female predominance was not seen in patients without IH on CT scan images. The difference was significant in the 51-60 and $61-70$ years' groups. ${ }^{*} \mathrm{p}<0.05,{ }^{* *} \mathrm{p}<0.01$ 


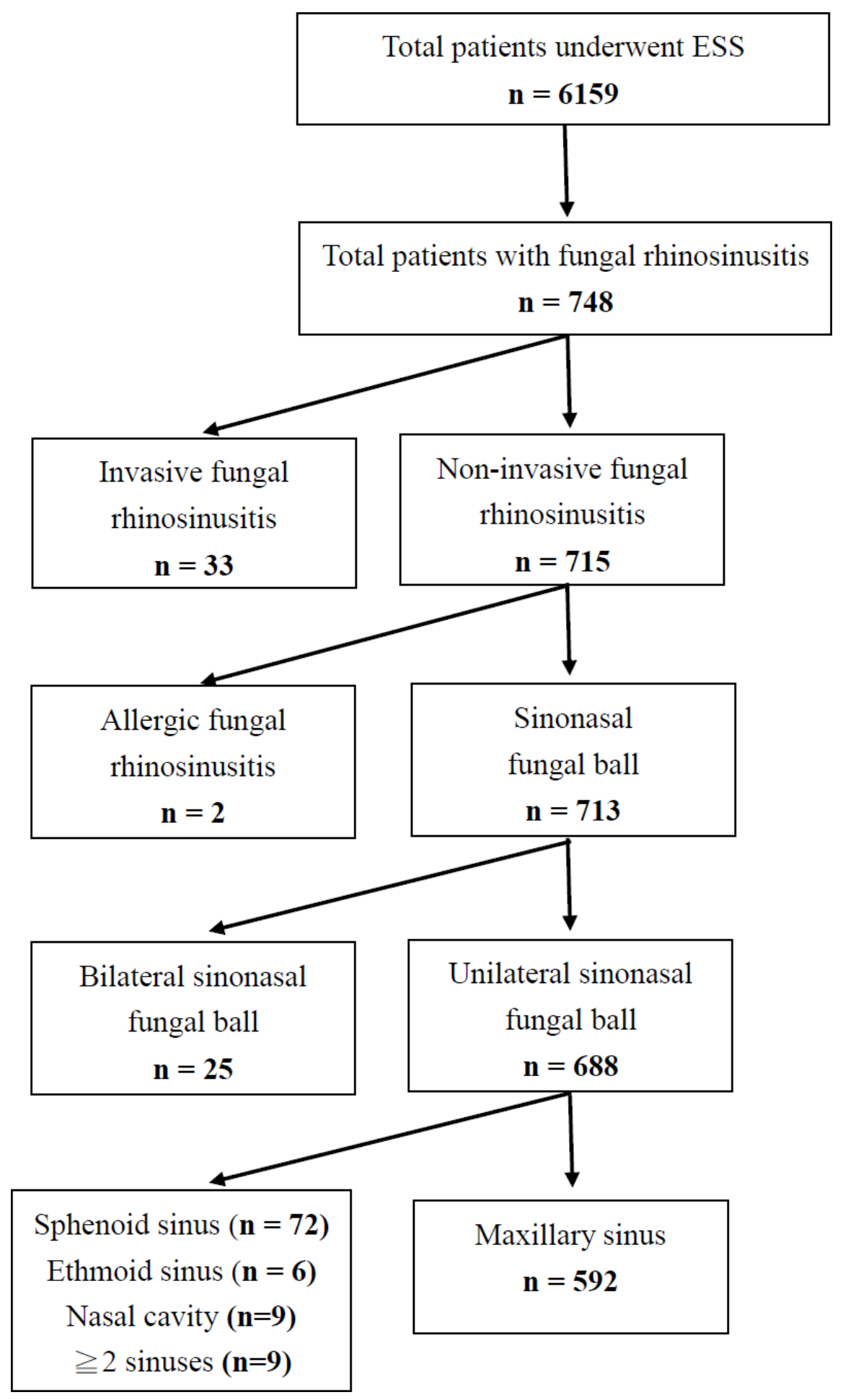



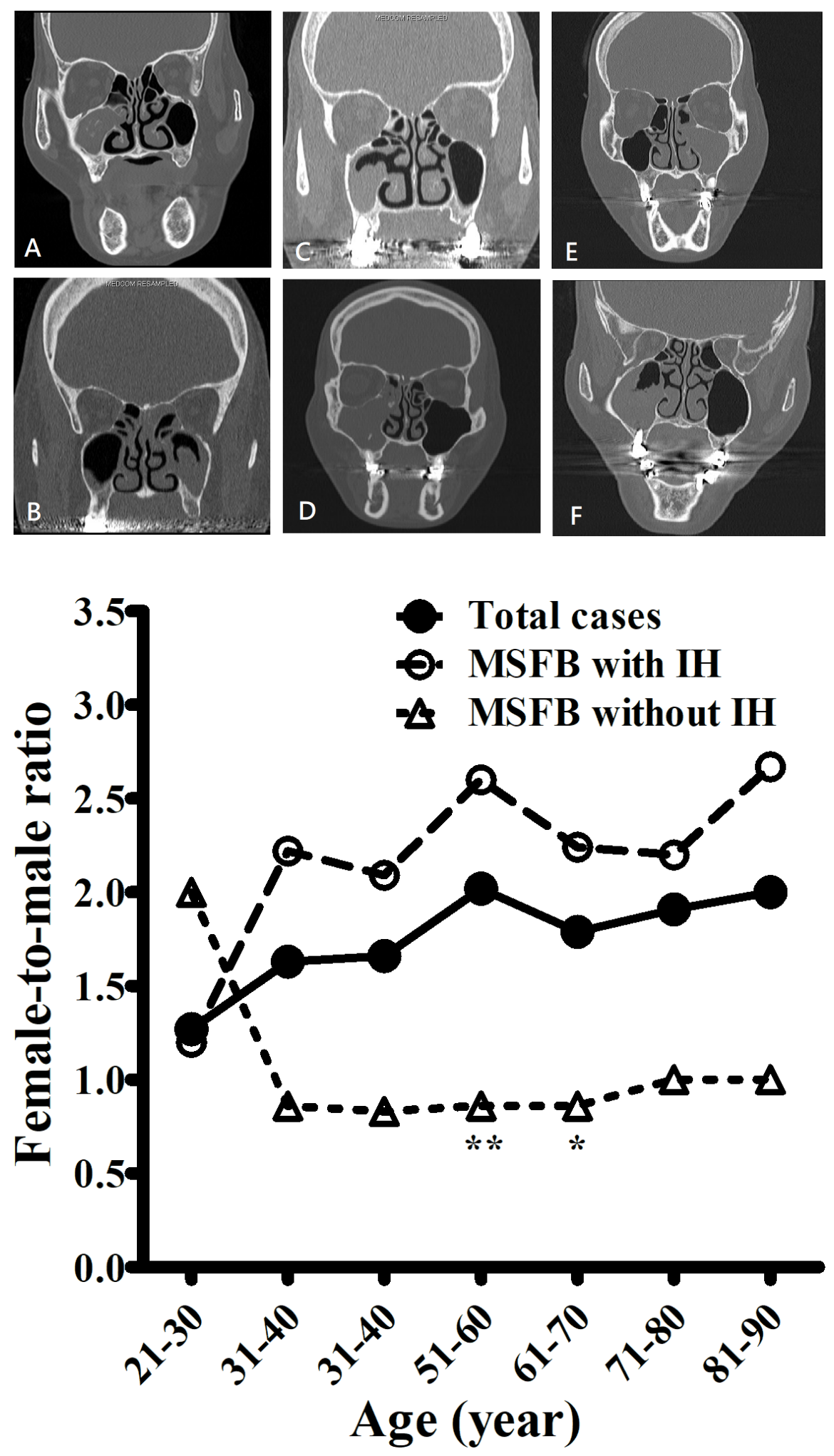